Bull. Soc. math. France

133 (1), 2005, p. 73-86

\title{
EXISTENCE DE FILTRATIONS ADMISSIBLES SUR DES ISOCRISTAUX
}

\author{
par Jean-Marc Fontaine \& Michael Rapoport
}

\begin{abstract}
RÉsumé. - Soit $(D, \varphi)$ un isocristal de vecteur de Newton $\nu \in\left(\mathbb{Q}^{d}\right)_{+}$. On associe à une filtration $\mathcal{F}^{\bullet}$ de $D$ son vecteur de Hodge $\mu\left(\mathcal{F}^{\bullet}\right) \in\left(\mathbb{Z}^{d}\right)_{+}$. Si $\mathcal{F}^{\bullet}$ est admissible (i.e. $\left(D, \varphi, \mathcal{F}^{\bullet}\right)$ est faiblement admissible en tant qu'isocristal filtré), alors $\mu\left(\mathcal{F}^{\bullet}\right) \geq \nu$. Réciproquement, on démontre qu'étant donné $\mu \in\left(\mathbb{Z}^{d}\right)_{+}$avec $\mu \geq \nu$, il existe une filtration admissible $\mathcal{F}^{\bullet}$ de $D$ avec $\mu=\mu\left(\mathcal{F}^{\bullet}\right)$. On en déduit, à l'aide d'un théorème de Laffaille, l'existence d'un réseau $M$ dans $D$ de type $\mu$. On donne aussi une variante pour un groupe quasi-déployé quelconque.
\end{abstract}

AbStRact (Existence of admissible filtrations on isocrystals). - Let $(D, \varphi)$ be an $F$-isocrystal with associated Newton vector $\nu$ in $\left(\mathbb{Q}^{d}\right)_{+}$. To a filtration $\mathcal{F}^{\bullet}$ of $D$ is associated its Hodge vector $\mu\left(\mathcal{F}^{\bullet}\right) \in\left(\mathbb{Z}^{d}\right)_{+}$. If $\mathcal{F}^{\bullet}$ is admissible $\left(\right.$ i.e. $\left(D, \varphi, \mathcal{F}^{\bullet}\right)$ is a weakly admissible filtered isocrystal), then $\mu\left(\mathcal{F}^{\bullet}\right) \geq \nu$. We show that, conversely, for any $\mu \in\left(\mathbb{Z}^{d}\right)_{+}$with $\mu \geq \nu$, there exists an admissible filtration $\mathcal{F}^{\bullet}$ of $D$ with $\mu=\mu\left(\mathcal{F}^{\bullet}\right)$. With the help of a theorem of Laffaille we deduce the existence of a lattice $M$ in $D$ of type $\mu$. We also give a variant for arbitrary quasi-split groups.

Texte reçu le 11 octobre 2002, accepté le 9 mai 2003

Jean-Marc Fontaine, Institut Universitaire de France et UMR 8628 du CNRS, Université de Paris-Sud, Mathématique, Bât. 425, 91405 Orsay Cedex (France)

E-mail : fontaine@math.u-psud.fr • Url : www.math.u-psud.fr

Michael RAPOPORT, Mathematisches Institut der Universität Bonn, Beringstr. 1, 53115

Bonn (Deutschland) • E-mail : rapoport@math.uni-bonn.de • Url : www.math.uni-

bonn.de

Classification mathématique par sujets (2000). — 14F30, 14L05.

Mots clefs. - Isocristaux, vecteur de Newton, filtrations admissibles.

BULLETIN DE LA SOCIÉTÉ MATHÉMATIQUE DE FRANCE

(C) Société Mathématique de France

$0037-9484 / 2005 / 73 / \$ 5.00$ 


\section{0. Énoncé des résultats}

Soit $F$ une extension finie de $\mathbb{Q}_{p}$. Soit $k$ un corps parfait de caractéristique $p$ contenant le corps résiduel $k_{F}$ de $F$. Notons $W(k)$ (resp. $W\left(k_{F}\right)$ ) l'anneau des vecteurs de Witt à coefficients dans $k$ (resp. $k_{F}$ ) et posons $L=F \otimes_{W\left(k_{F}\right)} W(k)$. C'est un corps, extension finie totalement ramifiée du corps des fractions de $W(k)$. Soient $q$ le cardinal de $k_{F}, \sigma_{0}$ l'automorphisme de $W(k)$ induit par fonctorialité par l'automorphisme $x \mapsto x^{q}$ sur $k$ et $\sigma$ l'automorphisme id $\otimes \sigma_{0}$ de $L$. Un isocristal (relativement $\grave{a}(F, k)$ ) est un espace vectoriel $D$ de dimension finie sur $L$, muni d'un endomorphisme $\varphi$ bijectif et $\sigma$-linéaire. Soit

$$
\left(\mathbb{Q}^{d}\right)_{+}=\left\{\left(\nu_{1}, \nu_{2}, \ldots, \nu_{d}\right) \in \mathbb{Q}^{d} ; \nu_{1} \geq \nu_{2} \geq \cdots \geq \nu_{d}\right\} .
$$

On associe à un isocristal $(D, \varphi)$ de dimension $d$ son vecteur de Newton $(=$ la suite des pentes avec multiplicités égales à la dimension de la composante isotypique correspondante, en ordre décroissant $), \nu(D, \varphi) \in\left(\mathbb{Q}^{d}\right)_{+}$. Soit

$$
\left(\mathbb{Z}^{d}\right)_{+}=\left(\mathbb{Q}^{d}\right)_{+} \cap \mathbb{Z}^{d} .
$$

Soit $\mu=\left(\mu_{1}, \ldots, \mu_{d}\right) \in\left(\mathbb{Z}^{d}\right)_{+}$. Soit $\mathcal{F}^{\bullet}$ une filtration de $D$ par des sous- $L$ espaces vectoriels, décroissante, exhaustive et séparée, indexée par $\mathbb{Z}$; on dit que $\mathcal{F}^{\bullet}$ est de type $\mu$ si

$$
\operatorname{dim} \operatorname{gr}_{i}^{\mathcal{F}^{\bullet}}(D)=\#\left\{j ; \mu_{j}=i\right\} .
$$

Autrement dit, les sauts de la filtration $\mathcal{F}^{\bullet}$ sont les $\mu_{j}(j=1, \ldots, d)$ et la taille du saut en $\mu_{j}$ est donnée par la multiplicité de $\mu_{j}$ dans $\mu$. Toute filtration $\mathcal{F}^{\bullet}$ a un type $\mu\left(\mathcal{F}^{\bullet}\right) \in\left(\mathbb{Z}^{d}\right)_{+}$bien déterminé.

Une filtration $\mathcal{F}^{\bullet}$ de l'isocristal $(D, \varphi)$ de dimension $d$ est dite admissible si les deux conditions suivantes sont satisfaites :

(i) en notant $|\nu|=\sum_{i=1}^{d} \nu_{i}$, pour $\nu=\left(\nu_{1}, \ldots, \nu_{d}\right) \in \mathbb{Q}^{d}$, on a

$$
\left|\mu\left(\mathcal{F}^{\bullet}\right)\right|=|\nu(D, \varphi)| ;
$$

(ii) soit $\left(D^{\prime}, \varphi^{\prime}\right)$ un sous-isocristal de $(D, \varphi)$ et soit $\mathcal{F}^{\bullet}$ la filtration induite $\operatorname{par} \mathcal{F} \bullet \operatorname{sur} D^{\prime}$; on a

$$
\left|\mu\left(\mathcal{F}^{\prime \bullet}\right)\right| \leq\left|\nu\left(D^{\prime}, \varphi^{\prime}\right)\right| .
$$

Remarques. - 1) Pour tout $\lambda=\left(\lambda_{1}, \lambda_{2}, \ldots, \lambda_{d}\right) \in\left(\mathbb{Q}^{d}\right)_{+}$, notons $P(\lambda)$ le polygone associé à $\lambda$, i.e. le polygone convexe (autrement dit, à pentes croissantes) du plan réel d'origine $(0,0)$ dont les pentes sont les $\lambda_{j}$, la longueur de la projection du segment de pente $\lambda_{j}$ sur l'axe des $x$ étant égale à la multiplicité de $\lambda_{j}$ dans $\lambda$. Le nombre rationnel $|\nu(D, \varphi)|$, noté $t_{N}(D)$ dans [3], est toujours un entier; les points de coordonnées $(0,0)$ et $(d,|\nu(D, \varphi)|)$ sont les extrémités du polygone $P(\nu(D, \varphi))$ (parfois appelé polygone de Newton de l'isocristal $(D, \varphi))$. De même, $\left|\mu\left(\mathcal{F}^{\bullet}\right)\right|$ est noté $t_{H}\left(\mathcal{F}^{\bullet}\right)$ dans [3]; les points de coordonnées 
$(0,0)$ et $(d,|\mu(\mathcal{F} \bullet)|)$ sont les extrémités du polygone $P\left(\mu\left(\mathcal{F}^{\bullet}\right)\right)$ (parfois appelé polygone de Hodge de la filtration).

2) Dans le cas où $F=\mathbb{Q}_{p}$, dire qu'une filtration est admissible signifie qu'elle est faiblement admissible au sens de [3]. Le résultat principal de [1] signifie qu'elle est alors également admissible au sens de [3]. Si $\bar{L}$ désigne une clôture algébrique de $L$, on dispose donc (loc.cit.) d'une équivalence naturelle entre la catégorie des isocristaux (relativement à $\left(\mathbb{Q}_{p}, k\right)$ ) munis d'une filtration admissible, et la catégorie des représentations $p$-adiques cristallines de $\operatorname{Gal}(\bar{L} / L)$.

3) Ce résultat s'étend au cas où $F$ est une extension finie arbitraire de $\mathbb{Q}_{p}$. Soit $C_{p}$ le complété de $\bar{L}$ pour la topologie $p$-adique. Appelons $F$-représentation de $\operatorname{Gal}(\bar{L} / L)$ la donnée d'un $F$-espace vectoriel de dimension finie $V$ muni d'une action linéaire et continue de $\operatorname{Gal}(\bar{L} / L)$. Pour une telle représentation, notons $V_{C_{p}, F}$ le noyau de l'application $C_{p}$-linéaire naturelle de $C_{p} \otimes_{\mathbb{Q}_{p}} V$ dans $C_{p} \otimes_{F} V$. On dispose alors d'une équivalence naturelle entre la catégorie des isocristaux (relativement à $(F, k)$ ) munis d'une filtration admissible et la sous-catégorie pleine de la catégorie des $F$-représentations de $\operatorname{Gal}(\bar{L} / L)$ dont les objets sont les $V$ qui sont cristallines en tant que représentations $p$-adiques et vérifient la condition

$$
\left\{\begin{array}{l}
\text { le } C_{p} \text {-espace vectoriel } V_{C_{p}, F} \text { est engendré } \\
\text { par les éléments fixes par } \operatorname{Gal}(\bar{L} / L) .
\end{array}\right.
$$

Lorsque $V$ provient d'un groupe formel $\Phi$, cette dernière condition signifie que $\Phi$ est un $O_{F}$-module formel ( $c f$. par exemple, [2]). Par exemple, soit $\pi$ une uniformisante de $F$ et soit $(D, \varphi)=(L, \pi \sigma)$; la seule filtration admissible est celle pour laquelle $\operatorname{gr}_{1}^{\mathcal{F}}(D) \neq 0$. La $F$-représentation de $\operatorname{Gal}(\bar{L} / L)$ associée est de dimension 1 et c'est la duale de la restriction à $\operatorname{Gal}(\bar{L} / L)$ de celle que fournit un groupe formel de Lubin-Tate pour $F$ correspondant à $\pi$. Soit $\eta$ : $\operatorname{Gal}(\bar{L} / L) \rightarrow F^{\times}$le caractère qui définit l'action du groupe de Galois. Si $\tau$ est un $\mathbb{Q}_{p}$-automorphisme non trivial de $F$, la $F$-représentation de dimension 1 définie par $\tau \eta$ est encore cristalline mais elle ne vérifie pas $\left(^{*}\right)$.

Les remarques 2) et 3) ne seront pas utilisées dans la suite.

Sur $\left(\mathbb{Q}^{d}\right)_{+}$, on dispose de l'ordre partiel pour lequel $\lambda \leq \lambda^{\prime}$ si

$$
\lambda_{1}+\lambda_{2}+\cdots+\lambda_{r} \leq \lambda_{1}^{\prime}+\lambda_{2}^{\prime}+\cdots+\lambda_{r}^{\prime}, \text { pour } r=1,2, \ldots, d-1,
$$

et $\lambda_{1}+\lambda_{2}+\cdots+\lambda_{d}=\lambda_{1}^{\prime}+\lambda_{2}^{\prime}+\cdots+\lambda_{d}^{\prime}$.

Dire que $\lambda \leq \lambda^{\prime}$ équivaut à dire que $P(\lambda)$ est au-dessus de $P\left(\lambda^{\prime}\right)$ et que ces deux polygones ont mêmes extrémités.

ThÉORÈme 1. - Supposons $k$ algébriquement clos. Soit $(D, \varphi)$ un isocristal de dimension $d$. Soit $\mu \in\left(\mathbb{Z}^{d}\right)_{+}$. Pour qu'il existe une filtration faiblement admissible de type $\mu$ sur $D$, il faut et il suffit que $\mu \geq \nu(D, \varphi)$. 
Soit $O_{L}$ l'anneau des entiers de $L$, et soit $\pi \in O_{F}$ une uniformisante qui est donc aussi une uniformisante de l'anneau de valuation discrète $O_{L}$. Soit $(D, \varphi)$ un isocristal de dimension $d$. Un $O_{L}$-réseau $M$ de $D$ est dit de type $\mu \in\left(\mathbb{Z}^{d}\right)_{+}$s'il existe une base $e_{1}, \ldots, e_{d}$ de $M$ tel que $\pi^{\mu_{1}} e_{1}, \ldots, \pi^{\mu_{d}} e_{d}$ soit une base de $\varphi(M)$. Tout $O_{L}$-réseau $M$ a un type bien déterminé que l'on note $\mu(M)$.

ThÉORÈme 2. - Supposons $k$ algébriquement clos. Soit $(D, \varphi)$ un isocristal de dimension d. Soit $\mu \in\left(\mathbb{Z}^{d}\right)_{+}$. Alors il existe un $O_{L}$-réseau de type $\mu$ dans $D$ si et seulement si $\mu \geq \nu(D, \varphi)$.

D'après [3, prop. 4.3.3], la condition d'admissibilité équivaut à demander que $P\left(\mu\left(\mathcal{F}^{\bullet}\right)\right)$ et $P(\nu(D, \varphi))$ ont mêmes extrémités et que, pour tout sous-isocristal $\left(D^{\prime}, \varphi^{\prime}\right)$ de $(D, \varphi)$, si $\mathcal{F}^{\bullet}$ est la filtration induite par $\mathcal{F}^{\bullet}$, alors $P\left(\mu\left(\mathcal{F}^{\bullet}\right)\right)$ est en dessous de $P\left(\nu\left(D^{\prime}, \varphi^{\prime}\right)\right)$. L'implication directe du théorème 1 en résulte.

L'implication directe du théorème 2 est l'inégalité de Mazur ( $c f .[5$, th. 1.4.1]). Le contenu de ces deux théorèmes est donc la réciproque à ces inégalités. Le lien entre eux est donné par un résultat de Laffaille [10], comme on va le voir au $\S 1$.

Le théorème 2 est aussi obtenu, avec une preuve différente, dans [9]. En fait, on a une version de l'inégalité de Mazur pour un groupe réductif quasidéployé dans $F$ et déployé dans une extension non ramifiée de $F(c f$. [12]). On peut conjecturer que la réciproque à cette inégalité est vraie, dans ce contexte (existence de certains sous-groupes parahoriques hyperspéciaux), et on peut la démontrer dans certains cas ( $c f$. . [11], [9]). En ce qui concerne la généralisation du théorème 1 dans cette direction, on a le résultat suivant :

Soit $G$ un groupe réductif connexe quasi-déployé sur $F$. Soit $A$ un tore déployé maximal. Soit $T$ le tore maximal contenant $A$. Fixons un sous-groupe de Borel $B$ contenant $T$. Soit $\bar{C}$ la chambre de Weyl fermée dans $X_{*}(T) \otimes \mathbb{R}$ correspondante. Si $\bar{F}$ est une clôture algébrique de $F$, le groupe $\Gamma=\operatorname{Gal}(\bar{F} / F)$ agit sur $\bar{C}$. Soient $\mathfrak{A}=X_{*}(A) \otimes \mathbb{Q}$ et $\mathfrak{A}_{+}=\mathfrak{A} \cap \bar{C}=\bar{C}^{\Gamma} \cap\left(X_{*}(T) \otimes \mathbb{Q}\right)$.

Soient $L^{\prime}$ une extension finie de $L$ et $\mu: \mathbb{G}_{m} \rightarrow G$ un morphisme défini sur $L^{\prime}$. On note $\mu_{0}$ l'unique conjugué de $\mu$ qui appartient à $\bar{C} \cap X_{*}(T)$. On pose

$$
\bar{\mu}=\frac{1}{\left(\Gamma: \Gamma_{\mu_{0}}\right)} \sum_{\tau \in \Gamma / \Gamma_{\mu_{0}}} \tau \mu_{0} \in \mathfrak{A}_{+} .
$$

C'est un élément de $\mathfrak{A}_{+}$qui ne dépend que de la classe de conjugaison $\{\mu\}$ de $\mu$.

Soit $b \in G(L)$. On note $\overline{\nu_{b}} \in \mathfrak{A}_{+}$son point de Newton (cf. [8], introduction et $\S 3.2$ ). Rappelons comment on peut le définir : soit $\mathbb{D}$ le groupe diagonalisable sur $F$ de groupe des caractères $\mathbb{Q}$; on a $\operatorname{Hom}(\mathbb{D}, T)=\mathfrak{A}$. À toute représentation rationnelle $(V, \varrho)$ de $G$ est associée un isocristal $(D, \varphi)$, avec $D=V \otimes_{F} L$ 
et $\varphi=\varrho(b) \cdot\left(\operatorname{id}_{V} \otimes \sigma\right)$. La décomposition par les pentes

$$
D=\bigoplus_{\alpha \in \mathbb{Q}} D_{\alpha}
$$

peut être considérée comme un morphisme $\nu_{\varrho}: \mathbb{D} \rightarrow \mathrm{GL}(V)$ défini sur $L$. Il existe un unique morphisme $\nu_{b}: \mathbb{D} \rightarrow G$ tel que $\nu_{\varrho}=\varrho \circ \nu_{b}$ pour tout $\rho$. Alors $\bar{\nu}_{b}$ est l'unique conjugué de $\nu_{b}$ sous l'action de $G(L)$ qui est dans $\mathfrak{A}_{+}$. Il ne dépend que de la classe de $\sigma$-conjugaison de $b$ (voir [8]).

On a par ailleurs sur $\mathfrak{A}_{+}$l'ordre partiel usuel, pour lequel $\lambda \leq \lambda^{\prime}$ si et seulement si $\lambda^{\prime}-\lambda$ est combinaison linéaire à coefficients $\geq 0$ des coracines simples relatives à $\bar{C}$.

Une paire $(b, \mu)$ formée d'un élément $b \in G(L)$ et d'un homomorphisme $\mu: \mathbb{G}_{m} \rightarrow G$ défini sur une extension finie $L^{\prime}$ de $L$ est dite admissible (appelée faiblement admissible dans [13, déf. 1.18]) si, pour toute représentation rationnelle $(V, \varrho)$ de $G$, l'isocristal associé $(D, \varphi)$, muni de la filtration $\mathcal{F}^{\bullet} \operatorname{sur} D \otimes_{L} L^{\prime}$ induite par $\varrho \circ \mu$, est admissible. Il suffit pour vérifier cette propriété de la tester sur une représentation fidèle.

ThÉORÈme 3. - Supposons $k$ algébriquement clos. Soit $G$ un groupe réductif connexe quasi-déployé sur $F$. Soient $b \in G(L), L^{\prime}$ une extension finie de $L$ et $\mu: \mathbb{G}_{m} \rightarrow G$ un morphisme défini sur $L^{\prime}$. Pour qu'il existe $\mu^{\prime} \in\{\mu\}$ défini sur $L^{\prime}$ et tel que la paire $\left(b, \mu^{\prime}\right)$ soit admissible il faut et il suffit que $\bar{\mu} \geq \bar{\nu}_{b}$.

Explicitons ce théorème lorsque $G=T$ est un tore. Dans ce cas, $\{\mu\}$ correspond à un seul élément $\mu \in X_{*}(T)$ et $\bar{\mu}$ est la moyenne sur l'orbite $\Gamma \cdot \mu$; l'ordre partiel est trivial et l'énoncé signifie que

$$
(b, \mu) \text { est admissible } \Longleftrightarrow \bar{\mu}=\bar{\nu}_{b}
$$

Ceci est exactement l'équivalence entre (i) et (iii) de la proposition 1.21 de [13]. Le théorème 3 est donc à la fois une généralisation de cette proposition, qui est le cas d'un tore, et du théorème 1 , qui est le cas de $\mathrm{GL}_{d}$.

Remarque. - Dans le théorème 3, soit $E \subset L^{\prime}$ le corps de définition de la classe de conjugaison $\{\mu\}$ de $\mu$. Alors $E$ est une extension finie de $F$ et $L^{\prime}$ contient $E L$. Inversement, étant données une classe de conjugaison $\{\mu\}$ définie sur $E$ et une extension $L^{\prime}$ de $E L$, il existe $\mu \in\{\mu\}$ défini sur $L^{\prime}$, cf. [6, $\left.\S 1.4 .3\right]$.

Nous remercions G. Laumon pour des discussions utiles.

Durant la préparation de ce travail, le deuxième auteur a bénéficié du soutien financier du Ministère de la Recherche et de la Fondation A. von Humboldt (prix Gay-Lussac/Humboldt) et aussi de l'hospitalité des Universités de Paris (Jussieu et Orsay).

BULLETIN DE LA SOCIÉTÉ MATHÉMATIQUE DE FRANCE 


\section{Démonstrations des théorèmes 1 et 2}

Comme on l'a dit dans l'introduction, il s'agit seulement de vérifier les implications réciproques. On fixe l'isocristal $(D, \varphi)$ de dimension $d$.

Soit $s \in \mathbb{N}$ tel que $s \cdot \nu(D, \varphi) \in \mathbb{Z}^{d}$. Soit $F^{\prime}=F_{s}$ l'extension de degré $s$ de $F$ contenue dans $L$. Pour tout nombre rationnel $\alpha$ tel que $s \alpha \in \mathbb{Z}$, soit

$$
V_{\alpha}=\left\{v \in D ; \varphi^{s}(v)=\pi^{s \alpha} v\right\} .
$$

Soit $V=\sum V_{\alpha}$. Alors on sait que $V$ est somme directe des $V_{\alpha}$ et que l'application naturelle $V \otimes_{F^{\prime}} L \rightarrow D$ est un isomorphisme. En plus, tout sousisocristal $\left(D^{\prime}, \varphi^{\prime}\right)$ de $(D, \varphi)$ est rationnel sur $F^{\prime}$, i.e. il existe un unique sous$F^{\prime}$-espace vectoriel $V^{\prime}$ de $V$ tel que $D^{\prime}=V^{\prime} \otimes_{F^{\prime}} L$.

On fixe $\mu \in\left(\mathbb{Z}^{d}\right)_{+}$. Les filtrations $\mathcal{F}^{\bullet}$ de type $\mu$ sur $V \otimes_{F^{\prime}} K$, pour $K$ extension de $F^{\prime}$ variable, forment l'ensemble des $K$-points d'une variété algébrique projective $\mathcal{F}=\mathcal{F}(V, \mu)$ sur $F^{\prime}$. En fait, $\mathcal{F}$ est une variété de drapeaux partiels de $V$ et est de la forme

$$
\mathcal{F}=G / P,
$$

où $G=\mathrm{GL}(V)$ et où $P$ est un sous-groupe parabolique de $G$.

Un point $x \in \mathcal{F}(L)$ sera dit Weil-générique relativement à $F^{\prime}$ si le point image du composé

$$
\operatorname{Spec} L \stackrel{x}{\longrightarrow} \mathcal{F} \times_{\text {Spec } F^{\prime}} \operatorname{Spec} L \longrightarrow \mathcal{F}
$$

est le point générique de $\mathcal{F}$.

Lemme 1.1. - (i) Il existe toujours des points $x \in \mathcal{F}(L)$ Weil-génériques relativement à $F^{\prime}$.

(ii) Un tel point n'est contenu dans aucune sous-variété propre de $\mathcal{F}$ définie sur $F^{\prime}$.

Démonstration. - (i) Par le lemme de Bruhat, on sait que le corps des fonctions $K$ de $\mathcal{F}$ est une extension transcendante pure de $F^{\prime}$. Comme $L$ est de degré de transcendance infinie sur $F^{\prime}, K$ peut être plongé dans $L$.

(ii) C'est évident.

Lemme 1.2. - Soit $\mathcal{F}^{\bullet}$ une filtration de $D$ de type $\mu$ correspondant à un point Weil-générique relativement à $F^{\prime}$ de $\mathcal{F}(L)$. Soit $\left(D^{\prime}, \varphi^{\prime}\right)$ un sous-isocristal de $(D, \varphi)$. Alors le type de la filtration $\mathcal{F}^{\prime \bullet}$ induite par $\mathcal{F}^{\bullet}$ sur $D^{\prime}$ est donné par

$$
\mu\left(\mathcal{F}^{\bullet \bullet}\right)=\left(\mu_{d-d^{\prime}+1}, \ldots, \mu_{d-1}, \mu_{d}\right), \quad \text { où } d^{\prime}=\operatorname{dim} D^{\prime} .
$$

Démonstration. - La filtration $\mathcal{F}^{\bullet}$ est transverse à $D^{\prime}$, puisque les filtrations qui ne le sont pas forment une sous-variété propre de $\mathcal{F}$ définie sur $F^{\prime}$ (le complément de l'unique orbite ouverte du parabolique standard de type $\left(d^{\prime}, d-d^{\prime}\right)$ 
sur $G / P)$. On a donc

$$
\operatorname{dim}\left(\mathcal{F}^{i} \cap D^{\prime}\right)=\max \left(0, \operatorname{dim} \mathcal{F}^{i}-\left(d-d^{\prime}\right)\right), \quad \forall i \in \mathbb{Z},
$$

et ceci implique l'assertion.

Démonstration du théorème 1. - Soient donc $\mu \geq \nu(D, \varphi)$ et $\mathcal{F} \bullet$ une filtration de $D$ correspondant à un point Weil-générique relativement à $F^{\prime}$. Montrons que $\mathcal{F} \bullet$ est admissible. La condition (i) de l'admissibilité $|\mu(\mathcal{F} \bullet)|=|\nu(D, \varphi)|$ (cf. introduction) résulte de la définition même de l'ordre partiel sur $\left(\mathbb{Q}^{d}\right)_{+}$. Soit $\left(D^{\prime}, \varphi^{\prime}\right)$ un sous-isocristal de $(D, \varphi)$ de dimension $d^{\prime}$. Alors $\left(D^{\prime}, \varphi^{\prime}\right)$ est un facteur direct de $(D, \varphi)$ et son vecteur des pentes est de la forme

$$
\nu\left(D^{\prime}, \varphi^{\prime}\right)=\left(\nu_{j_{1}}, \ldots, \nu_{j_{d^{\prime}}}\right), \quad \text { pour } 1 \leq j_{1}<j_{2}<\cdots<j_{d^{\prime}} \leq d .
$$

D'après le lemme 1.2 , la filtration $\mathcal{F}^{\bullet} \bullet$ sur $D^{\prime}$ induite par $\mathcal{F} \bullet$ est de type $\mu\left(\mathcal{F}^{\prime \bullet}\right)=\left(\mu_{d-d^{\prime}+1}, \ldots, \mu_{d}\right)$. L'inégalité dans (ii) de la définition de l'admissibilité résulte alors de

$$
\sum_{i=1}^{d^{\prime}} \mu_{d-d^{\prime}+i} \leq \sum_{i=1}^{d^{\prime}} \nu_{d-d^{\prime}+i} \leq \sum_{i=1}^{d^{\prime}} \nu_{j_{i}} .
$$

Le théorème 2 résulte alors de la proposition suivante (pour laquelle il n'est pas nécessaire de supposer $k$ algébriquement clos) :

Proposition 1.3. - Soient $(D, \varphi)$ un isocristal de dimension d et $\mu \in\left(\mathbb{Z}^{d}\right)_{+}$. Les assertions suivantes sont équivalentes:

(i) il existe une filtration admissible $\mathcal{F}^{\bullet}$ de type $\mu$ sur $D$,

(ii) il existe un $O_{L}$-réseau de type $\mu$.

Démonstration. - C'est une conséquence du résultat de Laffaille [10] : soit $(D, \varphi)$ un isocristal et soit $\mathcal{F} \bullet$ une filtration de $D$. Rappelons ( $c f$. [10]) qu'un $O_{L}$-réseau $M$ dans $D$ est dit adapté à la filtration $\mathcal{F} \bullet$ (ou, dans une autre terminologie [4], que le réseau $M$, muni de sa filtration $\mathcal{F} \bullet \cap$, est fortement divisible), si l'on a

$$
M=\varphi\left(\sum_{i} \pi^{-i}\left(\mathcal{F}^{i} \cap M\right)\right) .
$$

D'après le théorème 3.2 de $[10]^{(1)}$, la filtration $\mathcal{F} \bullet$ est faiblement admissible si et seulement s'il existe un réseau $M$ adapté à $\mathcal{F}^{\bullet}$. La proposition résulte alors du lemme suivant :

(1) Stricto sensu, ce résultat n'est énoncé dans [10] que lorsque $F=\mathbb{Q}_{p}$ et la filtration est positive. Par une torsion à la Tate, on se ramène au cas d'une filtration positive. La preuve de Laffaille s'étend alors telle quelle au cas considéré ici : il suffit de remplacer $p$ par $\pi$.

BULLETIN DE LA SOCIÉTÉ MATHÉMATIQUE DE FRANCE 
Lemme 1.4. - Soit $(D, \varphi)$ un isocristal de dimension d et soit $\mathcal{F} \bullet$ une filtration de type $\mu\left(\mathcal{F}^{\bullet}\right)$ sur $D$. Soit $M$ un réseau adaptée à $\mathcal{F}^{\bullet}$. Alors

$$
\mu(M)=\mu\left(\mathcal{F}^{\bullet}\right) .
$$

Démonstration. - Soit $u_{1}, u_{2}, \ldots, u_{d}$ une base de $M$ sur $O_{L}$ adaptée à la filtration, i.e. telle que, si $\mu_{r}$ désigne le plus grand des entiers $i$ vérifiant $u_{r} \in \mathcal{F}^{i} \cap M$, pour $1 \leq r \leq d$, alors

$$
\mathcal{F}^{i} \cap M=\bigoplus_{\mu_{r} \geq i} O_{L} u_{r}
$$

Quitte à changer l'ordre des $u_{r}$, on peut supposer que $\mu_{1} \geq \mu_{2} \geq \cdots \geq \mu_{d}$ et on a alors $\mu\left(\mathcal{F}^{\bullet}\right)=\left(\mu_{1}, \mu_{2}, \ldots, \mu_{d}\right)$.

Si l'on pose $e_{r}=\pi^{-\mu_{r}} \varphi\left(u_{r}\right)$, on voit que $e_{1}, e_{2}, \ldots, e_{d}$ est une base de $M$ sur $O_{L}$ tandis que $\pi^{\mu_{1}} e_{1}, \pi^{\mu_{2}} e_{2}, \ldots, \pi^{\mu_{d}} e_{d}$ est une base de $\varphi(M)$. On a donc $\mu(M)=\left(\mu_{1}, \mu_{2}, \ldots, \mu_{d}\right)$.

Remarque. - Les énoncés des théorèmes 1 et 2 ne s'étendent pas au cas où le corps $k$ n'est plus algébriquement clos. Un exemple est donné par $L=F$, $(D, \varphi)=\left(L^{2}\right.$, id $), \mu=(r,-r)$ où $r$ est un entier $\geq 1$. Alors $\mu \geq(0,0)=\nu(D, \varphi)$, mais il n'existe pas de filtration admissible de type $\mu$.

\section{Démonstration du théorème 3}

Soient $G$ quasi-déployé sur $F, L^{\prime}$ une extension finie de $L$ et $\mu: \mathbb{G}_{m} \rightarrow G$ un morphisme défini sur $L^{\prime}$. Avec les notations de l'introduction, à $\mu$ correspondent des éléments $\mu_{0} \in \bar{C}$ et $\bar{\mu} \in \mathfrak{A}_{+}$qui ne dépendent que de la classe de conjugaison $\{\mu\}$ de $\mu$.

Soit $(V, \varrho)$ une représentation rationnelle de $G$, où $V$ est un espace vectoriel de dimension $d$ sur $F$. Alors on associe à $\{\mu\}$ une classe de conjugaison $\{\varrho \circ \mu\}$ de morphismes de $\mathbb{G}_{m}$ dans $\mathrm{GL}(V)$ à laquelle correspond un élément bien défini $\varrho\left(\mu_{0}\right)$ de $\left(\mathbb{Z}^{d}\right)_{+}$. De même, $\bar{\mu}$ et par ailleurs n'importe quel élément $\bar{\nu} \in \mathfrak{A}_{+}$ définissent des éléments $\varrho(\bar{\mu})$ et $\varrho(\bar{\nu})$ de $\left(\mathbb{Q}^{d}\right)_{+}$.

Lemme 2.1. - Soit $\bar{\nu} \in \mathfrak{A}_{+}$. Les conditions suivantes sont équivalentes :

(i) $\bar{\mu} \leq \bar{\nu}$ (dans l'ordre partiel sur $\mathfrak{A}_{+}$),

(ii) $\varrho(\bar{\mu}) \leq \varrho(\bar{\nu})$, pour toute représentation rationnelle $(V, \varrho)$ de $G$,

(ii') $\varrho\left(\mu_{0}\right) \leq \varrho(\bar{\nu})$, pour toute représentation rationnelle $(V, \varrho)$ de $G$,

(iii) comme en (ii), pour une représentation fidèle $(V, \varrho)$ de $G$,

(iii') comme en (ii'), pour une représentation fidèle $(V, \varrho)$ de $G$.

Démonstration. - On remarque que la classe de conjugaison $\{\varrho \circ \mu\}$ est définie sur $F$, de sorte que $\varrho\left(\mu_{0}\right)=\varrho(\bar{\mu})$. Ceci étant, l'assertion résulte de $[12, \S 2]$. 
Ce lemme entraîne déjà l'implication directe dans le théorème 3 , comme conséquence de l'implication directe dans le théorème 1.

Un cocaractère $f: \mathbb{G}_{m} \rightarrow G$ définit, sur chaque représentation linéaire $V$ de $G$, une graduation $V=\bigoplus_{n \in \mathbb{Z}} V_{n}$ donc aussi une filtration décroissante en posant

$$
\mathrm{Fil}^{r} V=\bigoplus_{n \geq r} V_{n}
$$

Deux cocaractères de $G$ sont dit par-équivalents s'ils définissent les mêmes filtrations sur les représentations linéaires de $V$. Les classes d'équivalence dans la classe de conjugaison $\{\mu\}$ de $\mu$ forment une variété projective $\mathcal{F}=\mathcal{F}(G,\{\mu\})$ définie sur $L^{\prime}$. On peut écrire

$$
\mathcal{F}(G,\{\mu\})=G_{L^{\prime}} / P_{\mu},
$$

où $P_{\mu}$ est un sous-groupe parabolique défini sur $L^{\prime}$.

Soit $b \in G(L)$. Pour démontrer le théorème 3 , on peut remplacer $b \in G(L)$ par un élément $\sigma$-conjugué $b^{\prime}=g b \sigma(g)^{-1}$, pour $g \in G(L)$, car $(b, \mu)$ est admissible si et seulement si $\left(g b \sigma(g)^{-1}, g \mu g^{-1}\right)$ l'est. D'après Kottwitz [7, $\left.\S 4\right]$, on peut donc supposer que $b$ vérifie une identité de décence (voir [13, p. 8]), c'est-à-dire qu'il existe un entier $s>0$ tel que

$$
(b \sigma)^{s}=\left(s \cdot \nu_{b}\right)(\pi) \sigma^{s}
$$

où $\nu_{b}: \mathbb{D} \rightarrow G$ a été défini avant l'énoncé du théorème 3 et $s$ désigne l'endomorphisme de $\mathbb{D}$ induit par la multiplication par $s$ sur $\mathbb{Q}$.

On associe à $x \in \mathcal{F}\left(L^{\prime}\right)$ son co-caractère canonique de Harder-Narasimhan

$$
\lambda_{x}: \mathbb{D} \longrightarrow G
$$

qui est bien défini (sur $L^{\prime}$ ) à par-équivalence près. Il est caractérisé par le fait que pour toute représentation rationnelle $(V, \varrho)$ de $G$ la $\mathbb{Q}$-filtration sur $V \otimes_{F} L$ induite par $\varrho \circ \lambda_{x}$ est la filtration canonique (indexée par les $H N$-pentes) de l'isocristal filtré $\left(V \otimes_{F} L, \varrho(b) \cdot(\right.$ id $\left.\otimes \sigma), \mathcal{F}_{\varrho(x)}^{\bullet}\right),(c f$. par exemple, [13, prop. 1.4]). Soit $F^{\prime}$ l'unique extension de degré $s$ de $F$ contenue dans $L$. On sait que $\lambda_{x}$ est défini sur $F^{\prime}$ (voir [13, prop. 1.36]).

Soit $G_{\mathrm{ab}}=G / G_{\mathrm{der}}$ le tore quotient maximal de $G$. L'isomorphisme

$$
\mathcal{F}(G,\{\mu\}) \stackrel{\sim}{\longrightarrow} \mathcal{F}\left(G_{\mathrm{ad}},\left\{\mu_{\mathrm{ad}}\right\}\right) \times \mathcal{F}\left(G_{\mathrm{ab}},\left\{\mu_{\mathrm{ab}}\right\}\right)
$$

induit une bijection entre points (faiblement) admissibles,

$$
\mathcal{F}(G,\{\mu\})\left(L^{\prime}\right)^{\mathrm{adm}} \stackrel{\sim}{\longrightarrow} \mathcal{F}\left(G_{\mathrm{ad}},\left\{\mu_{\mathrm{ad}}\right\}\right)\left(L^{\prime}\right)^{\mathrm{adm}} \times \mathcal{F}\left(G_{\mathrm{ab}},\left\{\mu_{\mathrm{ab}}\right\}\right)\left(L^{\prime}\right)^{\mathrm{adm}} .
$$

Pour démontrer le théorème 3 , on peut donc supposer que $G$ est semi-simple, puisque le cas d'un tore est déjà réglé par [13, prop. 1.21], comme on l'a expliqué dans l'introduction.

BULLETIN DE LA SOCIÉTÉ MATHÉMATIQUE DE FRANCE 
Soit donc $G$ semi-simple et soit $E^{\prime}$ une extension finie de $F^{\prime}$ contenue dans $L^{\prime}$ sur laquelle la classe de conjugaison de $\mu$ est définie. Soit $x \in \mathcal{F}\left(L^{\prime}\right)$ Weilgénérique relativement à $E^{\prime}$. De tels points existent d'après le lemme 1.1 (lemme de Bruhat pour $G$ ). Montrons que $x$ est faiblement admissible sous l'hypothèse $\bar{\mu} \geq \bar{\nu}_{b}$. Il suffit de voir que le cocaractère $\lambda_{x}$ associé à $x$ est trivial. Raisonnons par l'absurde et supposons que le parabolique $P_{x}=P_{\lambda_{x}}$ soit propre. Ce parabolique est défini sur $F^{\prime}$.

Lemme 2.2. - Soient $G$ un groupe quasi-déployé sur $F$ et $B$ un sous-groupe de Borel défini sur F. Soit $P$ un sous-groupe parabolique propre défini sur $F^{\prime}$.

(i) Il existe une représentation rationnelle irréductible $(V, \varrho)$ de $G$ et une droite $\ell \subset V \otimes_{F} F^{\prime}$, telles que $P=\operatorname{Stab}_{G}(\ell)$.

(ii) Pour une telle représentation, soit

$$
W_{\bullet}=\left((0) \subset W_{1} \subset W_{2} \subset \cdots \subset W_{d}=V\right)
$$

un drapeau complet de $V$ stable par $\varrho(B)$. Alors il existe $g \in G\left(F^{\prime}\right)$ tel que $\ell$ soit transverse à $\varrho(g) \cdot W_{\bullet}$, i.e., tel que $\ell \not \subset \varrho(g) W_{d-1}$.

Démonstration. - (i) On peut évidemment supposer que $P$ est standard, i.e. contient $B \otimes_{F} F^{\prime}$. Soit $\bar{C}^{*} \subset X^{*}(T) \otimes \mathbb{R}$ la chambre de Weyl fermée dans l'espace des caractères correspondant à $B$. Alors $P$ correspond à une facette de $\bar{C}^{*}$. Soit $\lambda \in X^{*}(T)$ à l'intérieur de cette facette et soit $V_{\lambda}$ la représentation irréductible de plus haut poids $\lambda$. Alors $V_{\lambda}$ est un espace vectoriel sur $F^{\prime}$ sur lequel agit $G_{F^{\prime}}$. Soit $F_{\lambda}=F^{\Gamma_{\lambda}}$. Alors $V_{\lambda}$ est défini sur $F_{\lambda}$, i.e. est de la forme $V_{\lambda}=V_{\lambda}^{0} \otimes_{F_{\lambda}} F^{\prime}$ et $V_{\lambda}^{0}$ est irréductible comme représentation de $G_{F_{\lambda}}$. Le $F$-espace vectoriel cherché $V$ est égal à $V_{\lambda}^{0}$ et est muni de la représentation rationnelle $\varrho$ de $G$ définie par la composition des homomorphismes canoniques

$$
G \longrightarrow R_{F_{\lambda} / F}\left(G_{F_{\lambda}}\right) \longrightarrow R_{F_{\lambda} / F}\left(\operatorname{GL}_{F_{\lambda}}\left(V_{\lambda}^{0}\right)\right)=\operatorname{GL}_{F}(V)
$$

(cf. [14, th. 7.2]). Alors $(V, \varrho)$ répond à la question en prenant pour $\ell$ la droite engendré par un vecteur de poids $\lambda$ dans $V_{\lambda}$.

(ii) Le sous-espace de $V \otimes_{F} F^{\prime}$ engendré par $\left\{\varrho(g) x ; g \in G\left(F^{\prime}\right), x \in \ell\right\}$ est une représentation irréductible de $G_{F^{\prime}}$. Le plus petit sous-espace défini sur $F$ et le contenant est une représentation de $G$. Son espace ne peut donc pas être contenu dans $W_{d-1}$.

Appliquons ce lemme au parabolique $P_{\lambda_{x}}$. On considère l'isocristal

$$
(D, \varphi)=\left(V \otimes_{F} L, \varrho(b) \cdot(\operatorname{id} \otimes \sigma)\right)
$$

muni de sa filtration $\mathcal{F}_{\varrho(x)}^{\bullet}$ sur $D \otimes_{L} L^{\prime}$. Soit $D^{\prime}=\ell \otimes_{F^{\prime}} L$. Alors $D^{\prime}$ est un cran dans la filtration de Harder-Narasimhan de $D$, car il est stable par le parabolique de Harder-Narasimhan $P_{\varrho \circ \lambda_{x}}$ de $\mathrm{GL}(V)_{L}$. Le type $\mu\left(\mathcal{F}^{\mathbf{\bullet}}\right) \in \mathbb{Z}$ de la 
filtration induite par $\mathcal{F}_{\varrho(x)}$ sur $D^{\prime} \otimes_{L} L^{\prime}$ et la pente $\nu\left(D^{\prime}, \varphi^{\prime}\right) \in \mathbb{Q}$ de l'isocristal $\left(D^{\prime}, \varphi^{\prime}\right)=\left(D^{\prime}, \varphi_{\mid D^{\prime}}\right)$ vérifient donc

$$
\mu\left(\mathcal{F}^{\bullet \bullet}\right)>\nu\left(D^{\prime}, \varphi^{\prime}\right)
$$

Le lemme 2.2 implique que les points $y \in \mathcal{F}\left(L^{\prime}\right)$ tels que la filtration $\mathcal{F}_{\varrho}{ }^{\prime}(y)$ de $D \otimes_{L} L^{\prime}$ ne soit pas transverse à $D^{\prime} \otimes_{L} L^{\prime}$ forment une sous-variété fermé propre de $\mathcal{F}$ défini sur $E^{\prime}$. Comme $x$ est Weil-générique relativement à $E^{\prime}$, il ne peut pas être contenu dans cette sous-variété. Soit $\mathcal{F}_{\varrho(x)}$ de type $\left(\mu_{1}, \ldots, \mu_{d}\right) \in\left(\mathbb{Z}^{d}\right)_{+}$. Comme dans le lemme 1.2, la transversalité implique que

$$
\mu\left(\mathcal{F}^{\bullet}\right)=\mu_{d}
$$

Soit $\nu(D, \varphi)=\left(\nu_{1}, \ldots, \nu_{d}\right) \in\left(\mathbb{Q}^{d}\right)_{+}$. Alors $\nu\left(D^{\prime}, \varphi^{\prime}\right)=\nu_{j}$, pour un $j$ avec $1 \leq j \leq d$. Comme $\bar{\mu} \geq \bar{\nu}_{\bar{b}}$, le lemme 2.1 montre que $\left(\mu_{1}, \ldots, \mu_{d}\right) \geq\left(\nu_{1}, \ldots, \nu_{d}\right)$, ce qui nous donne

$$
\mu\left(\mathcal{F}^{\prime \bullet}\right)=\mu_{d} \leq \nu_{d} \leq \nu_{j}=\nu\left(D^{\prime}, \varphi^{\prime}\right)
$$

contredisant (2.6).

\section{Remarques supplémentaires}

Supposons $k$ algébriquement clos.

a) D'après la proposition 1.3 , dans le cas de $\mathrm{GL}_{d}$, les deux théorèmes d'existence sont équivalents. Il faut pourtant souligner que dans le contexte d'un groupe réductif $G$, quasi-déployé sur $F$ et déployé sur une extension non ramifiée de $F$, l'existence d'un sommet hyper-spécial d'un type $\mu$ implique, outre l'inégalité $\bar{\mu} \geq \bar{\nu}_{b}$ qui apparaît dans le théorème 3 , l'identité

$$
\mu^{\natural}=\kappa(b)
$$

dans $\pi_{1}(G)_{\Gamma}(c f .[12, \S 4])$. On voit donc qu'a priori, dans le cas général, la question d'existence de réseaux est plus subtile que la question d'existence de filtrations. Le cas de $\mathrm{GL}_{d}$ est exceptionnel car alors (3.1) résulte de l'inégalité $\bar{\mu} \geq \bar{\nu}_{b}$. Le lien entre le théorème 1 et le théorème 2 se fait via le théorème de Laffaille sur l'existence de réseaux fortement divisibles et on peut se demander s'il existe une notion analogue en théorie de groupes.

b) La construction de Laffaille d'un réseau adapté à une filtration admissible fournit à partir d'un réseau $M$ arbitraire un unique réseau adapté maximal $M^{\text {max }}$ contenu dans $M$, et aussi un unique réseau adapté minimal $M^{\text {min }}$ contenant $M$. Cette construction n'est malheureusement pas compatible aux opérations d'algèbre linéaire usuelles (produit tensoriel : $\left(M_{1} \otimes M_{2}\right)^{\max } \neq$ $M_{1}^{\max } \otimes M_{2}^{\max },\left(M_{1} \otimes M_{2}\right)^{\min } \neq M_{1}^{\min } \otimes M_{2}^{\min } ;$ passage au dual : on a $\left(M^{*}\right)^{\max }=\left(M^{\min }\right)^{*}$ et non $\left.\left(M^{\max }\right)^{*}\right)$. Ceci, tout comme la remarque précédente, semble montrer qu'il n'est pas possible de déduire du théorème 2 et du théorème 3 pour le groupe symplectique l'existence de réseaux autoduaux 
d'un type donné dans un isocristal symplectique (un tel théorème d'existence est pourtant démontré dans [9] par d'autres méthodes).

c) On peut s'intéresser à un problème beaucoup plus général que celui auquel les théorèmes 1 et 3 fournissent une réponse. Explicitons dans le cas de $\mathrm{GL}_{d}$. Soit $(D, \varphi)$ un isocristal de dimension $d$. Soit $\mu \in\left(\mathbb{Z}^{d}\right)_{+}$avec $|\mu|=|\nu(D, \varphi)|$, et soit $\mathcal{F}$ la variété des filtrations de type $\mu$ sur $D$. Soit $x \in \mathcal{F}(L)$ et soit $\lambda_{x}: \mathbb{D} \rightarrow \mathrm{GL}(D)$ le $H N$-cocaractère associé (bien défini à par-équivalence près). Alors $\lambda_{x}$ définit un élément bien déterminé $\lambda(x) \in\left(\mathbb{Q}^{d}\right)_{+}$avec $|\lambda(x)|=0$. On obtient ainsi une décomposition disjointe

$$
\mathcal{F}(L)=\bigcup_{\lambda \in\left(\mathbb{Q}^{d}\right)_{+},|\lambda|=0}^{\bullet} \mathcal{F}(L)_{\lambda},
$$

où $\mathcal{F}(L)_{\lambda}=\{x \in \mathcal{F}(L) ; \lambda(x)=\lambda\}$.

Si $\mu \geq \nu(D, \varphi)$, le théorème 1 montre que l'unique élément minimal $\lambda=0$ de l'ensemble d'indices donne une contribution non vide à (3.2). Même dans le cas $\mu \geq \nu(D, \varphi)$, nous n'avons pas déterminé l'ensemble des indices dans (3.2) avec une contribution non vide. Si $\mu \nsupseteq \nu(D, \varphi)$, nous ignorons même les indices minimaux parmi ceux avec une contribution non vide à (3.2).

d) Rappelons que $C_{p}$ désigne le complété d'une clôture algébrique de $L$. Soient $G, b, L^{\prime}, \mu$ comme dans l'énoncé du théorème 3. Alors on sait [13, prop. 1.36], que les points admissibles par rapport à $b$ dans $\mathcal{F}(G,\{\mu\})\left(C_{p}\right)$ forment un espace rigide-analytique sur $L^{\prime}$, ouvert admissible de $\mathcal{F}(G,\{\mu\})$, que nous noterons $\mathcal{F}(G,\{\mu\})^{\mathrm{adm}}$. On peut alors reformuler ainsi le théorème 3 :

ThÉorème $3^{\prime}$. - On suppose $k$ algébriquement clos. Soit $G, b, L^{\prime}, \mu$ comme dans le théorème 3 . Les conditions suivantes sont équivalentes:

(i) $\mathcal{F}(G,\{\mu\})^{\mathrm{adm}} \neq \varnothing$,

(ii) $\mathcal{F}(G,\{\mu\})^{\operatorname{adm}}\left(L^{\prime}\right) \neq \varnothing$,

(iii) $\bar{\mu} \geq \bar{\nu}_{b}$.

En effet, (i) $\Rightarrow$ (iii) se démontre comme dans le $\S 2$ par réduction à l'inégalité $[3, \S 4.3 .3]$ pour le cas de $\mathrm{GL}_{d}$. L'implication (iii) $\Rightarrow$ (ii) est le théorème 3 et l'implication (ii) $\Rightarrow$ (i) est triviale.

e) Soit $(D, \varphi, N)$ un $(\varphi, N)$-module sur $L$ (cf. par exemple, [1]), c'est-à-dire un isocristal muni d'une application $L$-linéaire $N: D \rightarrow D$ telle que $N \varphi=$ $q \varphi N$ (où, rappelons-le, $q$ est le nombre d'éléments du corps résiduel de $F$ ). On a encore une notion de filtration admissible dans ce cadre et le théorème 1 s'étend : cela résulte de ce que toute filtration admissible sur l'isocristal sousjacent (obtenu en oubliant l'action de $N$ ) est a fortiori admissible sur le $(\varphi, N)$ module. 


\section{BIBLIOGRAPHIE}

[1] Colmez (P.) \& Fontaine (J.-M.) - Construction des représentations semi-stables, Invent. Math., t. 140 (2000), pp. 1-43.

[2] Dechunert (J.-M.) - Classification des A-modules formels, C. R. Acad. Sci. Paris Sér. I Math., t. 282 (1976), pp. 1413-1416.

[3] Fontaine (J.-M.) - Modules galoisiens, modules filtrés et anneaux de Barsotti-Tate, in Journées de Géométrie algébrique de Rennes (juillet 1978), III, Astérisque, vol.65, Société Mathématique de France, 1979, pp. 3-80.

[4] Fontaine (J.-M.) \& Laffaille (G.) - Construction de représentations p-adiques, Ann. Sci. École Norm. Sup., t. 15 (1982), pp. 547-608.

[5] Katz (N.) - Slope filtration of F-crystals, in Journées de Géométrie algébrique de Rennes (juillet 1978), I, Astérisque, vol.63, Société Mathématique de France, 1979, pp. 113-163.

[6] Kottwitz (R.) - Shimura varieties and twisted orbital integrals, Math. Ann., t. 269 (1984), pp. 287-300.

[7] _ Isocrystals with additional structure, Comp. Math., t. 56 (1985), pp. 201-222.

[8] _ Isocrystals with additional structure. II, Comp. Math., t. 109 (1997), pp. 255-339.

[9] Kottwitz (R.) \& Rapoport (M.) - On the existence of F-crystals, Comment. Math. Helv., t. 78 (2003), pp. 153-184.

[10] Laffaille (G.) - Groupes p-divisibles et modules filtrés : le cas peu ramifié, Bull. Soc. Math. France, t. 108 (1980), pp. 187-206.

[11] Rapoport (M.) - A positivity property of the Satake isomorphism, Manuscripta Math., t. 101 (2000), pp. 153-166.

[12] RApoport (M.) \& RichaRtz (M.) - On the classification and specialization of F-isocrystals with additional structure, Comp. Math., t. 103 (1996), pp. 153-181.

[13] Rapoport (M.) \& Zink (Тн.) - Period spaces for p-divisible groups, Annals of Mathematics Studies, vol. 141, Princeton University Press, 1996.

[14] Tits (J.) - Représentations linéaires irréductibles d'un groupe réductif sur un corps quelconque, J. reine angew. Math., t. 247 (1971), pp. 196-220.

\section{Note ajoutée sur épreuves (octobre 2003)}

Voici une liste de travaux récents concernant le cercle de problèmes abordés dans cet article.

Kottwitz (R.) - On the Hodge-Newton decomposition for split groups, Internat. Math. Res. Notices, t. 26 (2003), pp. 1433-1447. 
LeIGH (C.) - A converse to Mazur's inequality for split classical groups, math. NT/0211327.

ORLIK (S.) - On Harder-Narasimhan strata in flag manifolds, math.NT/ 0304222 .

Wintenberger (J.-P.) - Existence de F-cristaux avec structures supplémentaires, math.NT/0303026. 